\title{
Influencia de la satisfacción y el valor percibido sobre el "Word of Mouth" en los usuarios de centros deportivos
}

\section{Influence of the satisfaction and the perceived value related to the "Word of Mouth" in costumers of sports center}

\author{
Fernando García Pascual*, Núria Molina García y Javier Mundina Gómez
}

Universitat de Valencia (España)

\begin{abstract}
Resumen: Uno de los principales objetivos de los servicios deportivos, es fidelizar a los usuarios el mayor tiempo posible en dicho servicio. Por ello, desde el inicio de la literatura de la gestión del deporte, que analizan las diferentes variables de gestión, cada vez más, aparecen trabajos que tratan de aportar información relacionada con este objetivo. Dentro de las intenciones futuras de los usuarios, la recomendación es uno de los factores claves para poder analizar los futuros comportamientos de dichos usuarios. Por ello este trabajo, trata de analizar cuál es la influencia de la satisfacción y el valor percibido de los usuarios, sobre el "Word of Mouth" (boca-oído), es decir, como influyen estas dos variables en las futuras recomendaciones de los mismos usuarios. Para así, aportar a los diferentes servicios deportivos, y a los gestores del deporte en particular, información valiosa para obtener clientes fieles al servicio.

Palabras clave: Word of Mouth, satisfacción, valor percibido, centros deportivos.
\end{abstract}

Abstract: One of the main objectives of sports services is to retain users as long as possible in this service. For this reason, since the beginning of the literature on sports management, which analyzes the different management variables, more and more works appear that try to provide information related to this objective. Within the future intentions of the users, the recommendation is one of the main factors to be able to analyze the future behaviors of these users. For this reason, this research tries to analyze which is the influence of the satisfaction and the perceived value of the users, on the "Word of Mouth", that is, how these two variables influence the future recommendations of the same users. In this way, to provide different sports services, and sports managers in particular, valuable information to obtain loyal customers to the service.

Key words: Word of Mouth, satisfaction, perceived value, sports center.

\section{Introducción}

Vivimos en una sociedad, donde las empresas tratan de adueñarse de las diferentes competencias existentes en materia de mercado. Dentro del sector de los servicios deportivos, estas empresas tratan de convertir dicho servicio en una herramienta fiable y efectiva que permita retener y afianzar el binomio consumidor-empresa el mayor tiempo posible.

Actualmente existen multitud centros deportivos, dichos centros ofertan gran diversidad de programas y ofertas, para poder satisfacer a los usuarios del servicio, así como tratar de retener el mayor tiempo posible a estos usuarios. Por ello, las empresas, en concreto, los servicios deportivos, están en un continuo trabajo para poder formarse y realizar estrategias que sirvan para poder alcanzar dichos objetivos prioritarios en la gestión del deporte.

El objetivo de este trabajo trata de analizar, dentro del contexto de los centros deportivos, qué influencia tienen las distintas variables de gestión, como el valor percibido y la satisfacción, sobre los futuros comportamientos de los clientes acerca del servicio que se les ha prestado.

Dirección para correspondencia [Correspondence address]: Fernando García Pascual. Universitat de Valencia (España). E-mail: fer_garcia88@hotmail.com

\section{Satisfacción del usuario}

Durante los últimos años, dentro de la literatura del marketing empresarial y del contexto profesional de la gestión del deporte, la satisfacción del consumidor ha sido muy analizada (López, González \& Muñoz, 2014).

Dentro de la larga y extensa literatura de la gestión del deporte, muchos han sido los autores que han conceptualizado la satisfacción del consumidor dentro de sus diferentes trabajos (Mazursky \& Geva, 1989; Hogarth \& Einhorn, 1992; Tsiros \& Mittal, 2000).

Olsen et al., (2005), argumentan en su trabajo que la satisfacción del consumidor es entendida como una evaluación global o un estado de sentimientos hacia un producto o servicio. Calabuig et al. (2008) defienden que, desde hace ya un tiempo, la satisfacción, es una de las variables más estudiadas dentro del contexto de las organizaciones deportivas, ya que ofrece un resultado en el que el usuario, de manera subjetiva mide su experiencia con dicha organización (García, Cepeda \& Martín, 2012; Murray \& Howat, 2002).

De manera más específica, dentro del contexto de los servicios deportivos, en concreto, los centros deportivos, también han sido multitud los autores que han analizado dichas 
variables de gestión (Pedragosa \& Correia, 2009; Silla et al., 2014). Siendo la satisfacción del consumidor una de las más estudiadas (Sabiote, 2010; Yoshida \& James, 2010), ya que tener conocimiento de la opinión que tiene el cliente acerca del servicio, puede verse modificada por las diferentes experiencias vividas (satisfactorias o insatisfactorias). A su vez, la lealtad, puede verse adulterada como consecuencia de una experiencia insatisfactoria en el acto de consumo (Morales \& Hernández, 2004). En el estudio de Luna-Arocas (2004), defendían que, en los servicios deportivos, la satisfacción del usuario es un concepto integral de la eficaz gestión que tienen las empresas.

En los diferentes trabajos encontrados en la literatura, se ha ratificado que la satisfacción es un predictor fuertemente ligado a las intenciones futuras de los usuarios (Calabuig et al., 2012; Clemes, Brush \& Collins, 2011). De manera más específica, que la satisfacción es un antecedente de la lealtad, es una relación confirmada por los diferentes trabajos realizados por multitudes de autores a lo largo de los últimos años (Oliver, 1999; López, González \& Muñoz, 2014). Pero, sin embargo, ambos conceptos no hace mucho tiempo atrás, dentro del ambiente empresarial han llegado a ser utilizados de manera separada (O’Malley, 1998).

\section{Valor percibido del servicio}

Diferentes autores definen el valor percibido como la valoración que tiene el cliente de comparar entre los beneficios obtenidos de un servicio/producto y los costes o sacrificios percibidos (Bigné et al., 2000; Gale, 1994; Monroe, 1990; Zeithaml, 1988). El valor percibido es de tal importancia, que algunos autores recomiendan que las empresas deben ser proveedoras de valor con respecto a sus clientes/usuarios, ya que esta adaptación les hará diferenciarse de las demás organizaciones y con ello mejorar sus resultados (Callarisa et al., 2002; Reinartz \& Kumar, 2003).

A lo largo de la literatura, se ha debatido en la apreciación de la satisfacción como antecedente o como consecuencia del valor percibido (Sabiote, 2010). Gran cantidad de trabajos aceptan la segunda opción, valorando la satisfacción como consecuencia del valor percibido, dicho de otro modo, el valor percibido es un antecedente de la satisfacción del cliente. Al mismo tiempo, estas dos variables de gestión también son antecedentes directos de las intenciones futuras de comportamiento, y dicha relación se evidencia en multitud de trabajos (Brady et al., 2005; Lu et al., 2011).

Chang y Wang (2011), en su trabajo analizan la relación entre el valor percibido y la fidelidad de un servicio, argumentando que son dos variables fuertemente relacionadas, concluyendo así que cuanto mayor es la percepción del valor, mayor fidelidad conseguirá la empresa por parte de sus clientes.

\section{Word of mouth}

Una de las grandes preocupaciones de las empresas, es la lealtad del consumidor (Gázquez \& De Cannière, 2007). Dicha preocupación, la lealtad del usuario, obliga a las diferentes empresas a realizar estrategias para poder fidelizar a los consumidores del servicio y así retenerlos, ante otras estrategias de servicios similares, de tal modo que dichas estrategias sirvan un mejor servicio, tanto a los usuarios que llevan más tiempo, como para aquellas que acaban de adquirir el servicio (Molina, 2016).

Se trata de conseguir que los clientes estén satisfechos para alcanzar, por un lado, su fidelidad, y por otro sus recomendaciones a otros clientes potenciales. De este modo, se puede concluir que la lealtad del consumidor es el objetivo principal del marketing de relaciones, ya que un cliente fiel no sólo volverá a comprarnos la próxima vez que necesite un servicio, sino que además hablará bien de nosotros en su entorno (Buttle, 2009).

Dentro de los diferentes trabajos que analizan las intenciones futuras, cada vez se está analizando más una de las dimensiones que mide dichas intenciones, el "Word of Mouth" (WoM). Este concepto es ampliamente considerado como un factor influyente que repercute en el comportamiento del consumidor (Daugherty \& Hoffman, 2014). Martínez y Martínez (2009) argumentan que un "WOM" positivo no solo influencia en los consumidores fieles sino también en aquellos que ya no son usuarios.

El "WoM", según Litvin et al., (2008) se define como la comunicación entre los consumidores sobre un producto, servicio o empresa en la que las fuentes se consideran independientes de la influencia comercial.

En el campo del comportamiento del consumidor, algunos estudios previos han demostrado que los consumidores prestan más atención a la información negativa que a la positiva (Park \& Lee, 2009; Cheung \& Thadani, 2012). Ya investigaciones más actuales, han reseñado de manera más concreta, en el contexto del diseño de producto, que las evaluaciones de los consumidores están vinculadas a su disposición a difundir "WoM" sobre el producto (Homburg et al., 2005). Siguiendo en esta línea, Rosario et al., (2016), argumentan que el "WoM" es utilizado para diferentes propósitos del consumidor y distintas formas de dicho "WoM" tiene impacto en el éxito del producto.

\section{Método}

\section{Participantes}

La muestra obtenida para este estudio, pertenece a un centro deportivo situado en una localidad de la provincia de Valencia, que abrió sus puertas en Abril del 2011 y que está ubicada 
a $7 \mathrm{~km}$ del área metropolitana de Valencia. Se administraron 421 cuestionarios, de los cuales se descartaron 15 , siendo finalmente 406 cuestionarios cumplimentados por los usuarios de la instalación deportiva objeto de estudio, de los cuales 220 encuestados eran hombres y 186 eran mujeres. En la recogida de la muestra se llevó a cabo un método de muestreo no probabilístico de conveniencia.

\section{Instrumento}

El cuestionario que medía las percepciones de los usuarios de la instalación deportiva, ha sido diseñado por diversas escalas en función de la literatura, acerca del aspecto a evaluar. Cada una de estas escalas han sido adaptadas, para medir un aspecto relacionado con las distintas variables de gestión de los usuarios de la instalación deportiva. Las dimensiones que conforman este instrumento son: Satisfacción General (Hightower, Brady \& Baker, 2002), Valor Percibido (Sweeney \& Soutar, 2001), Intenciones futuras (Zeithaml, Berry \& Parasuraman, 1996). En el caso de la satisfacción de los usuarios estaba compuesto por 2 ítems, el valor percibido se dividió en tres subdimensiones, valor emocional (3), valor precio (2), valor social (2), y por último las intenciones futuras que medían el "Word of Mouth" compuesta por 3 ítems. Todas las respuestas eran de escala Likert, siendo el valor mínimo 1 (Totalmente desacuerdo) y el valor máximo 5 (Totalmente de acuerdo).

\section{Procedimiento}

El cuestionario se suministró en la propia instalación deportiva, entre 21 de Marzo de 2016 y el 26 de Mayo de 2016. Se suministró en el hall principal, ya que es el punto neurálgico de acceso al centro deportivo. Se realizó en diferentes franjas horarias para poder conocer las percepciones de todos los perfiles de usuarios que acuden a la instalación.

\section{Análisis de datos}

Para análisis y tratamiento de datos se ha utilizado el SPSS (Statistical Package for the Social Sciences, Versión 20), con licencia de la Universitat de València, también se realizó un análisis de fiabilidad, para poder confirmar la consistencia interna de las escalas mediante el cálculo estadístico del alpha de Cronbach, por último, se realizó un análisis factorial confirmatorio (AFC) para comprobar la validez del modelo me- diante el programa de modelos de ecuaciones estructurales EQS.

\section{Resultados}

\section{Fiabilidad y validez de las escalas}

Para conocer la fiabilidad que nos ofrece estas escalas que componen el instrumento, analizaremos el alpha de Cronbach, el coeficiente de fiabilidad compuesta (FC) y la varianza media extraída (AVE).

Tabla 1. Coeficientes alpha de Cronbach $(\alpha)$, fiabilidad compuesta (FC) y varianza media extraída (AVE) del modelo

\begin{tabular}{lccc}
\hline & $\alpha$ & FC & AVE \\
\hline Valor emocional &, 70 &, 75 &, 60 \\
Valor precio &, 78 &, 80 &, 66 \\
Valor social &, 72 &, 74 &, 59 \\
Satisfacción &, 80 &, 81 &, 68 \\
\hline "WoM" &, 85 &, 85 &, 66 \\
\hline
\end{tabular}

Como se puede observar en la tabla 1, la escala que medía la satisfacción del usuario obtuvo un alpha de ,80. Por otro lado las dimensiones que evaluaban el valor percibido del servicio obtuvieron valores alpha de cronbach favorables, el valor emocional $(, 75)$, el valor precio $(, 80)$ y el valor social $(, 74)$. Por último, la escala que medía las intenciones futuras del usuario a través del "WoM" resultó un alpha de ,85. Como se observa se da una buena consistencia interna en las 5 escalas, ya que superan el punto de corte $(, 70)$ sugerido por Hair, Black, Babin, Anderson y Tatham (2006).

En cuanto a la fiabilidad compuesta (FC) se observa como los valores de las cinco escalas están comprendidos por encima de .70 valor que confirman en su trabajo Hair et al., (2006). Por tanto, se confirma la fiabilidad de las escalas utilizadas en el instrumento.

Así también, observando la varianza media extraída (AVE) de las escalas, se confirma que todas ellas tienen valores superiores a ,50 (Fornell \& Larcker, 1981).

Una vez comprobada la fiabilidad de nuestras escalas, para comprobar los índices de bondad de ajuste del modelo se realizó un análisis factorial confirmatorio (AFC), como se observa en la siguiente tabla 2 .

Tabla 2. Índices de ajuste del modelo predictivo.

\begin{tabular}{lccccccc}
\hline Modelo & $x^{2}$ & $\mathrm{gl}$ & $x^{2 /} \mathrm{gl}$ & RMSEA & NNFI & CFI & IFI \\
\hline $\begin{array}{l}5 \text { factores } \\
12 \text { ítems }\end{array}$ & 60.334 & 34 & 1,77 & .037 & .98 & .99 & .99 \\
\hline
\end{tabular}


El modelo final que se representa, obtiene un chi-cuadrado significativo $\left(x^{2}=60.334 ; \boldsymbol{g} l=34 ; \mathrm{p}<, 01\right)$ así como también se obtiene un chi-cuadrado normado que muestra un buen ajuste $\left(x^{2} / \boldsymbol{g} \boldsymbol{l}=1,77\right)$, ya que obtiene un valor por debajo de tres, punto de corte que sugirieron diversos autores (Bollen, 1989; Kline, 2005).También se observa como el error de Aproximación Cuadrático Medio (RMSEA), obtiene un valor de ajuste correcto de, 037 valor por debajo de ,08 punto de corte que refleja en su trabajo Hu y Bentler (1999). Así como los índices de ajuste, nos dan valores por encima de 0,90 (Hu \& Bentler, 1999), el índice de ajuste comparativo $(\mathrm{CFI}=99)$, el índice de ajuste no normalizado $(\mathrm{NNFI}=, 98)$ y el índice de ajuste incremental (IFI=,99).

Para poder dibujar el modelo y conocer las relaciones causales que se dan en el mismo, como se puede observar en la figura 1, se establecieron las ecuaciones estructurales de dicho modelo.

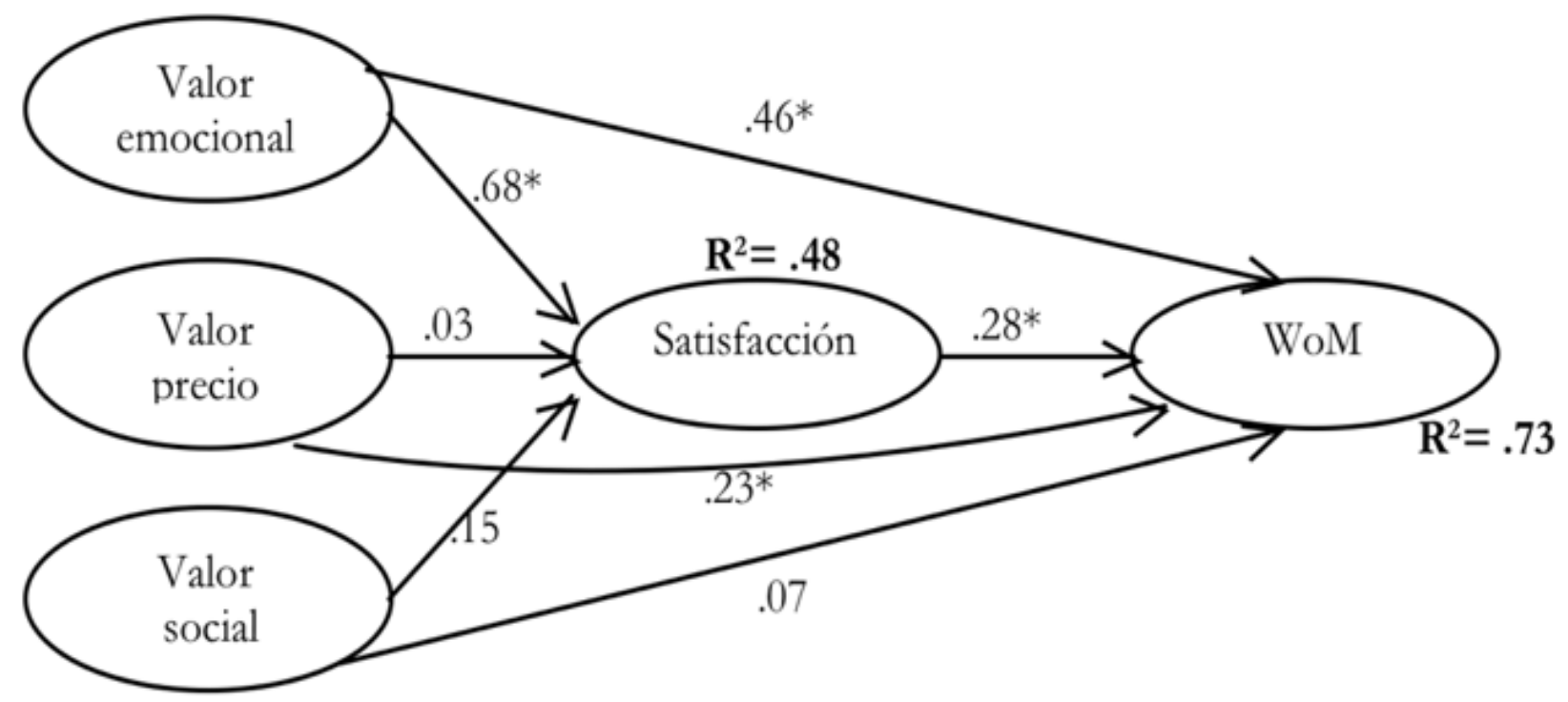

Figura 1. Modelo de relaciones causales del Word of Mouth (WoM).

En esta figura se observa como únicamente el valor emocional predice significativamente la satisfacción $(\beta=.68)$, explicando el $48 \%$ de la varianza. Por otro lado, y de forma sorprendente ni el valor precio, ni el valor social muestran significatividad sobre la satisfacción. En cuanto al "WoM", es predicho significativamente por la satisfacción $(\beta=.28)$, el valor precio $(\beta=.23)$ y el valor emocional $(\beta=.46)$ explicando el $73 \%$ de la varianza.

\section{Discusión y conclusión}

Dentro de este modelo, el valor percibido del servicio predice la satisfacción del usuario, dicha relación es ratificada por multitud de trabajos encontrados en la literatura (Cronin et al., 2000; Sánchez et al., 2006; Gil \& González, 2008; Ruiz-Alejos 2015). De manera general, dentro del ámbito de las empresas de servicios, existen trabajos que argumentan la labor del valor percibido como mediador de manera directa con la satisfacción (Brady et al., 2005; Lu et al., 2011), de manera más específica, dentro de los servicios deportivos también existen trabajos que corroboran dicha relación (Murray \& Howat, 2002). En sentido contrario, y mediante el trabajo desarrollado en Espańa, por Nuviala et al. (2015) dentro de los servicios deportivos españoles, no manifiestan apenas relación entre ambos conceptos de satisfacción y valor percibido, ya que el efecto del valor sobre la satisfacción resultó no significativa, y explicaba únicamente el 2\% de la varianza.

En nuestro modelo, de manera más segregada y en función de las tres dimensiones que construyen el valor percibido en este trabajo, se observa como de dichas tres dimensiones, solamente el valor emocional $(\beta=.68)$ predice significativamente la satisfacción, representando así un $48 \%$ de la varianza. Entendiendo así, que cuanto más se trabaje los estados emocionales y afectivos en dicho centro, mayor será la satisfacción de los usuarios de la instalación deportiva, coincidiendo así con los trabajos de Pedragosa et al. (2015) y Silla et al. (2014), en los que centran la atención en centros deportivos fitness, en la relación de las emociones con la satisfacción y las intenciones futuras. En concreto el trabajo de Pedragosa et al. (2015) acerca de la relación de las emociones y la satisfacción de los usuarios de centros deportivos, argumentan que las emociones, tanto positivas como negativas, tienen una influencia significativa sobre la satisfacción, y que dichas 
emociones positivas son las que influyen positivamente sobre la satisfacción del usuario (López-Torres et al., 2015).

Por otro lado, ni el valor precio ni el valor social, muestran significatividad con la satisfacción de los usuarios. Y al igual que el estudio de Silla et al. (2015) en centros deportivos, la dimensión valor precio no es significativa sobre la satisfacción de los usuarios de la instalación deportiva. Entendiendo que estos resultados se pueden dar por varios factores, en cuanto al valor precio, dicha instalación deportiva, tiene gran variedad de tarifas y precios que permiten acceder a todos los perfiles sociodemográficos de la sociedad, y de la población en particular, por ello los usuarios de la instalación pueden entender que el precio es ajustado y razonable con el valor de mercado de las instalaciones deportivas más cercanas a esta, y en consecuencia no influye en la satisfacción de los mismos. Por otro lado, el valor social, tampoco muestra significatividad con la satisfacción, hecho que puede ocurrir porque en el cuestionario, los indicadores que miden dicho valor están orientados a medir como es percibido el usuario por los demás cuando acude a la instalación, entendemos que no muestra significatividad porque al ser una instalación deportiva de población, donde los usuarios en su gran mayoría son de dicha localidad y se conocen entre ellos, hecho que evidencia que el usuario no le dé importancia a cómo es percibido por los demás usuarios, por lo tanto, dicho valor social no influye en si un usuario estará más o menos satisfecho con el servicio ofrecido.

Otra de los resultados observables en este modelo, son las relaciones de las tres dimensiones de valor percibido del servicio y de la satisfacción del usuario con las intenciones futuras, en nuestro caso con el "Word of Mouth". Se observa como el valor emocional $(\beta=.46)$, la satisfacción de los usuarios $(\beta$ $=.28)$ y el valor precio $(\beta=.23)$ predicen significativamente el "WoM", siendo el valor emocional la variable con más peso predictivo, todo ello explica el 73\% del "WoM". Por lo tanto, cuanto más positivas sean sus percepciones emocionales y de precios, así como más satisfecho este el cliente, más posibilidades existirán de que los usuarios recomienden la instalación deportiva. Estas relaciones también se han dado en otros trabajos encontrados en la literatura existente, Molina et al. (2016) en su trabajo que analizaba el efecto de las emociones en las intenciones futuras de los usuarios de los centros deportivos, también encontraron que el valor emocional era el que mayor predicción otorgaba a las intenciones futuras. Por otro lado, en el trabajo de Silla et al. (2015) que analiza los predictores de "WoM" en usuarios de centros deportivos, encuentran que el valor precio no predice significativamente el "WoM". Sin embargo, en el modelo que se analiza en este trabajo se encuentra como el valor social no predice el "WoM", dicho resultado puede darse al igual que ocurría con la satisfacción, porque entienden que acudir a esta instalación deportiva no influye en el modo en que es percibido por los demás usuarios.

Por tanto, observando el modelo, destacar que el valor emocional, no solamente predice de manera significativa la satisfacción y el "WoM", sino que es la que mayor peso ofrece en ambas dimensiones. De ahí la importancia y la significación que está obteniendo, en los últimos años, el estudio de las emociones en el deporte y en el contexto que lo envuelve. Dentro de estos trabajos, que miden las emociones y el deporte, existen diferentes ámbitos que han sido los más analizados, los espectadores de eventos deportivos (Calabuig et al., 2015; Calabuig, et al, 2016; Oshimi et al., 2014; Yoshida, James \& Cronin, 2013) organizaciones deportivas y tipos de servicios (Bigné et al., 2008; Molina, 2016).

\section{Limitaciones y futuras líneas de investigación}

Dentro del trabajo, encontramos la evaluación de los comportamientos conscientes de los usuarios de la instalación deportiva, es por ello la complejidad existente en tener una perspectiva racional y concreta de la respuesta que dará el usuario, en función de sus intenciones futuras con el servicio. Así también en la medición del valor percibido, una de las dimensiones que mayor protagonismo tiene, el valor emocional, también es complicado medirlo a través de preguntas y en un momento en el que no se adquiere el servicio.

En cuanto a las futuras líneas de investigación, quizá poder realizar este trabajo en diferentes tipologías de centros deportivos y en diferentes contextos, nos llevaría a poder ver las relaciones de todas las dimensiones de valor percibido con la satisfacción y el "WoM", ya que, en este trabajo, por ejemplo, el valor social no predice ninguna de las dos.

\section{Referencias}

1. Bigné, J. E., Moliner, M. A. y Callarisa, L. J. (2000). El valor y la fidelización de clientes: una propuesta de modelo dinámico de comportamiento. Revista Europea de Dirección y Economía de la empresa, 9 (3), 65-78.

2. Bigné, E., Matilla, A. y Andreu, L. (2008). The impact of experiential consumption cognitions and emotions on behavioral intentions. Journal of Services Marketing, 22 (4), 303-315.

3. Bollen, K. A. (1989). Structural equations with latent variables. New York: John Wiley y Sons.
4. Brady, M., Knight, G. A., Cronin, J., Hult, G. y Keilor, B. (2005). Removing the contextual lens: A multinational, multi-setting comparison of service evaluation models. Journal of Retailing, 81(3), 215-230.

5. Buttle, F. (2009). Customer Relationship Management: Concepts and Technologies. Burlington, MA: Butterworth-Heinemann.

6. Calabuig, F., Quintanilla, I., y Mundina, J. (2008). La calidad percibida de los servicios deportivos: diferencias según instalación, género, edad y tipo de usuario en según instalación, género, edad y tipo de usuario en servicios náuticos. Revista Internacional de Ciencias del Deporte, 
4(10), 25-43.

7. Calabuig, F., Molina, N. y Núnez, J. (2012). Una aplicación inicial del modelo tridimensional de calidad de servicio en centros deportivos privados. E-balonmano.com: Journal of Sport Science, 8 (1), 67-81.

8. Calabuig, F., Prado-Gascó, V., Crespo, J., Núñez, J. y Añó, V. (2015). Spectator emotions: Effects on quality, satisfaction, value and future intentions. Journal of Business Research, 68, 1445-1449.

9. Calabuig, F., Crespo, J., Núnez-Pomar, J., Valantine, I. y StaskeviciuteButiene, I. (2016). Role of perceived value and emotions in the satisfaction and future intentions of spectators in sporting events. Inzinerine Ekonomika-Engineering Economics, 27(2), 221-229.

10. Callarisa, L. J., Moliner, M. A., y Rodríguez, R. M. (2002). El componente emocional del valor percibido: un estudio cualitativo. XIV Encuentro de Profesores Universitarios de Marketing. Granada, 169-180.

11. Chang, H. y Wang, H. (2011). The moderating effect of customer perceived value on online shopping behaviour. Online Information Review, 35(3),333-359.

12. Cheung C. M., Thadani D. R. (2012). The impact of electronic wordof-mouth communication: a literature analysis and integrative model. Decis. Support Syst. 54, 461-470.

13. Clemes, M. D., Brush, G.J., y Collins, Mark J., 2011. Analysing the professional sport experience: A hierarchical approach. Sport Management Review, Elsevier, 14(4), 370-388.

14. Cronin, J.; Brady, M. y Hult, T. (2000). Assessing the effects of quality, value and customer satisfaction on consumer behavioral intentions in service environments, Journal of Retailing, 76 (2), 193-218.

15. Daugherty T., Hoffman E. (2014). eWOM and the importance of capturing consumer attention within social media. J. Mark. Commun. 20, 82-102.

16. Fornell, C. y Larcker, D. F. (1981). Evaluating structural equation models with unobservable variables and measurement error. Journal of marketing research, 39-50.

17. Gale, B. T. (1994). Managing Customer Value. New York: The Free Press.

18. García, J., Cepeda, G. y Martín, D. (2012). La satisfacción de clientes y su relación con la percepción de calidad en Centro de Fitness: utilización de la escala CALIDFIT. Revista de Psicología del Deporte, 21, 309-319.

19. Gázquez, J. C. y Decanniére, M. H. (2007). Impacto promocional vs relacional del mailing directo en elcomportamiento de compra del consumidor: una aplicación en el contexto de establecimientos de prendasde vestir. XIX Encuentro de Profesores Universitarios de Marketing.Vigo. ESIC, Madrid, 2-23.

20. Gil, I. y González, M. (2008). La investigación en valor percibido desde el marketing. Innovar, 18(31) 9-18.

21. Hightower, R., Brady, M. K. y Baker, T. L. (2002). Investigating the role of the physical environment in hedonic service consumption: An exploratory study of sporting events. Journal of Business Research. 55, 697-707.

22. Hogarth, R., y Einhorn, H. (1992). Order effects in belief updating: The belief-adjustment model. Cognitive Psychology, 24(1), 1-55.

23. Homburg, C., Koschate, N. y Hoyer, W.D. (2005), Do satisfied customers really pay more? A study of the relationship between customer satisfaction and willingness to pay. Journal of Marketing, 69 (2), 84-96.

24. Hu, L., y Bentler, P. M. (1999). Cutoff criteria for fit indexes in covariance structure analysis: Conventional criteria versus new alternatives. Structural Equation Modeling: A Multidisciplinary Journal, 6(1), 1-55.

25. Kline, R.B. (2005). Principles and practice of structural equation modeling (2nd ed.). New York: The Guilford Press.

26. Litvin S. W., Goldsmith R. E., Pan B. (2008). Electronic word-ofmouth in hospitality and tourism management. Tour. Manage. 29, 458-468.

27. López-Torres, M., Torregrosa, M. y Roca, R. (2007). Características de "Flow", ansiedad y estado emocional, en relación con el rendimiento de deportistas de élite. Cuadernos de Psicología de Deporte, 7(1), 25-44.

28. López, M.J., González, E. y Muñoz, P. (2014). La lealtad del consumidor en términos de calidad y satisfacción: análisis empírico en el sector de la banca on-line. Prospéritas, 1 (1), 57-67.

29. Lu, T., Tu, R., y Jen, W. (2011). The role of service value and switching barriers in an integrated model of behavioural intentions. Total Quality Management, 22(10), 1071-1089.

30. Luna-Arocas, R. (2004). Sistemas para medir y mejorar la satisfacción de los clientes en un centro deportivo. Curso sobre La gestión del deporte a través de la calidad. Albacete: Consejería de Cultura. Junta de Comunidades de Castilla-La Mancha.

31. Martínez, J. A. y Martínez, L. (2009). Gestión de clientes de servicios deportivos; un modelo de dinámica de sistemas. Revista Internacional de Medicina y Ciencias de la Actividad Física y el Deporte, 9 (36), 431 453.

32. Mazursky, D., y Geva, A. (1989). Temporal decay in satisfaction - Purchase intention relationship. Psychology y Marketing, 6(3), 211-227.

33. Molina, N. (2016). Evaluación de la calidad de servicio de centros deportivos privados. Influencia de la gestión en el bienestar de los usuarios. Tesis doctoral. Universitat de València.

34. Monroe, K. (1990). Pricing: Making Profitable Decisions, New York, McGraw Hill. Monroe, K. y Chapman

35. Morales V. y Hernández A., (2004). Calidad y satisfacción en los servicios: conceptualización. Efdeportes.com. Buenos Aires. 10, (73).

36. Murray, D. y Howat, G. (2002). The relationships among the Service Quality, Value, Satisfaction, and future intentions of Customers at an Australian Sports and Leisure Centre. Sport Mangement Review, 5, 25 43.

37. Nuviala, A., Grao-Cruces, A., Pérez-Ordás, R., Boceta. M., Nuviala, R. y González, J.A. (2012). Calidad, satisfacción y valor percibido de los usuarios de un servicio deportivo público. Movimento, 18(4), 11-32.

38. O’Malley, L. (1998). Can loyalty schemes really build loyalty?. Marketing Intelligence y Planning, 16 (1), 47-55.

39. Oliver, R.L. (1999) When consumer loyalty? Journal of Marketing, 63 , $33-44$.

40. Olsen, S.O., Wilcox, J. y Olsson, U. (2005). Consequences of ambivalence on satisfaction and loyalty. Psychology y Marketing, 22, (3), 247269.

41. Oshimi, D., Harada, M. y Fukuhara, T. (2014). Spectators' Emotions during Live Sporting Events: Analysis of Spectators after the Loss of the Supported Team at the 2013 FIFA Confederations Cup. Football Science, 11, 48-58.

42. Park C., Lee T. M. (2009). Information direction, website reputation and eWOM effect: a moderating role of product type. J. Bus. Res. 62, 61-67.

43. Pedragosa, V., Biscaia, R. y Correia, A. (2015). The role of emotions on consumers' satisfaction within the fitness context. Motriz, 21, 116- 124.

44. Pedragosa, V. y Correia, A. (2009). Expectations, satisfaction and loyalty in health and fitness clubs. International Journal of Sport Management and Marketing, 5, 450-464.

45. Reinartz, W. J. y Kumar, V. (2003). The impact of customer relationship characteristics on profitable lifetime duration. Journal of Marketing, 67(1), 77-99.

46. Rosario, A., Sotgiu, F., De Valck, K., y Bijmolt, T. (2016). The effect of electronic word of mouth on sales: A meta-analytic review of platform, product, and metric factors. Journal of Marketing Research, 53 (3), 297-229.

47. Ruíz-Alejos, C. (2015). Análisis de la calidad percibida, satisfacción, valor percibido e intenciones futuras de los usuarios de los servicios deportivos públicos gestionados por Logroño Deporte. Tesis Doctoral. Logroño: Universidad de la Rioja.

48. Sabiote, C. (2010). Valor percibido global del proceso de decisión de 
compra online de un producto turístico efecto moderador de compra. (Tesis doctoral). Universidad de Granada.

49. Sánchez, J., Callarisa, L. J., Rodríguez, R. M. y Moliner, M. A. (2006). Perceived value of the purchase of a tourism product. Tourism Management, 27(4), 394-409.

50. Silla, A., Calabuig, F. y Añó-Sanz, V. (2014). Emociones, satisfacción e intenciones futuras de los usuarios de actividades dirigidas de un centro deportivo. Journal of Sports Economics y Management, 4(1), 22-38.

51. Silla, A., Pérez, C. y Alonso, M. (2015). Predictores de WoM en usuarios de centros deportivos. VI Congreso Iberoamericano Economía del Deporte. Servicio de publicaciones. Universidad de Murcia.

52. Sweeney, J.C. y Soutar, G. (2001). Consumer perceived value: the development of a multiple item scale, Journal of Retailing, 77, 203-220.

53. Tsiros, M. y Mittal, V. (2000). Regret: a model of its antecedents and consequences in onsumer decision making, Journal of Consumer Research, 26 (4), 401-417.

54. Yoshida, M. y James, J.D. (2010). Customer satisfaction with game and service experiences: Antecedents and consequences. Journal of Sport Management, 24, 338-361.

55. Yoshida, M., James, J. y Cronin, J. (2013). Value creation: assessing the relationships between quality, consumption value and behavioral intentions at sporting events. International Journal of Sports Marketing and Sponsorship, 14(2), 51-73.

56. Zeithaml, V. (1988). Consumer perceptions of price, quality, and value: a means-end model and synthesis of evidence, Journal of Marketing, 52, $2-22$.

57. Zeithaml, V. A., Berry, L. L. y Parasuraman, A. (1996). The behavioral consequences of service quality. Journal of Marketing, 60 (2), 31-46. 